\title{
Außenstereotype über die deutsche Sprache und was sich dahinter verbirgt
}

,[...] il buio echeggiò di ordini stranieri, e di quei barbarici latrati dei tedeschi quando comandano, che sembrano dare vento a una rabbia vecchia di secoli““ (Primo Levi 1979:16). ${ }^{1}$

\section{Einleitung}

\subsection{Worum es geht}

Dass das Deutsche etwa „die raue Seite der europäischen Sprachlandschaft“ darstellt (Eichinger 2017:33) ${ }^{2}$, ist eine Meinung, die im europäischen Raum (sowohl unter Laien als auch unter Sprachwissenschaftler*innen ${ }^{3}$ ) sehr verbreitet zu sein scheint, und der sich Germanist*innen meist auch bewusst sind. Die zwei (stereo) typischen Einschätzungen, die dabei vielleicht am meisten herausstechen, sind die von der Härte der deutschen Sprache (meist mit dem Werturteil des Deutschen als einer hässlich klingenden Sprache verbunden) und die von der Schwierigkeit des

\footnotetext{
1 „,...] das Dunkel hallte wider von fremden Befehlen, jenem barbarischen Gebell kommandierender Deutscher, die sich eines jahrhundertealten Ingrimms zu entledigen scheinen." (Levi 2010:17) Dieser Satz kommt in dem autobiographischen Werk „Ist das ein Mensch?“ vor, in dem der italienische Chemiker und Schriftsteller Primo Levi über seine Zeit im Konzentrationslager Auschwitz berichtet.

2 „Die negativen Eigenschaften, die der deutschen Sprache und ihren Sprechern zugeschrieben werden, überwiegen“" (Stickel 2003:10).

3 Vgl. Trudgill/Giles (1976:7).
} 


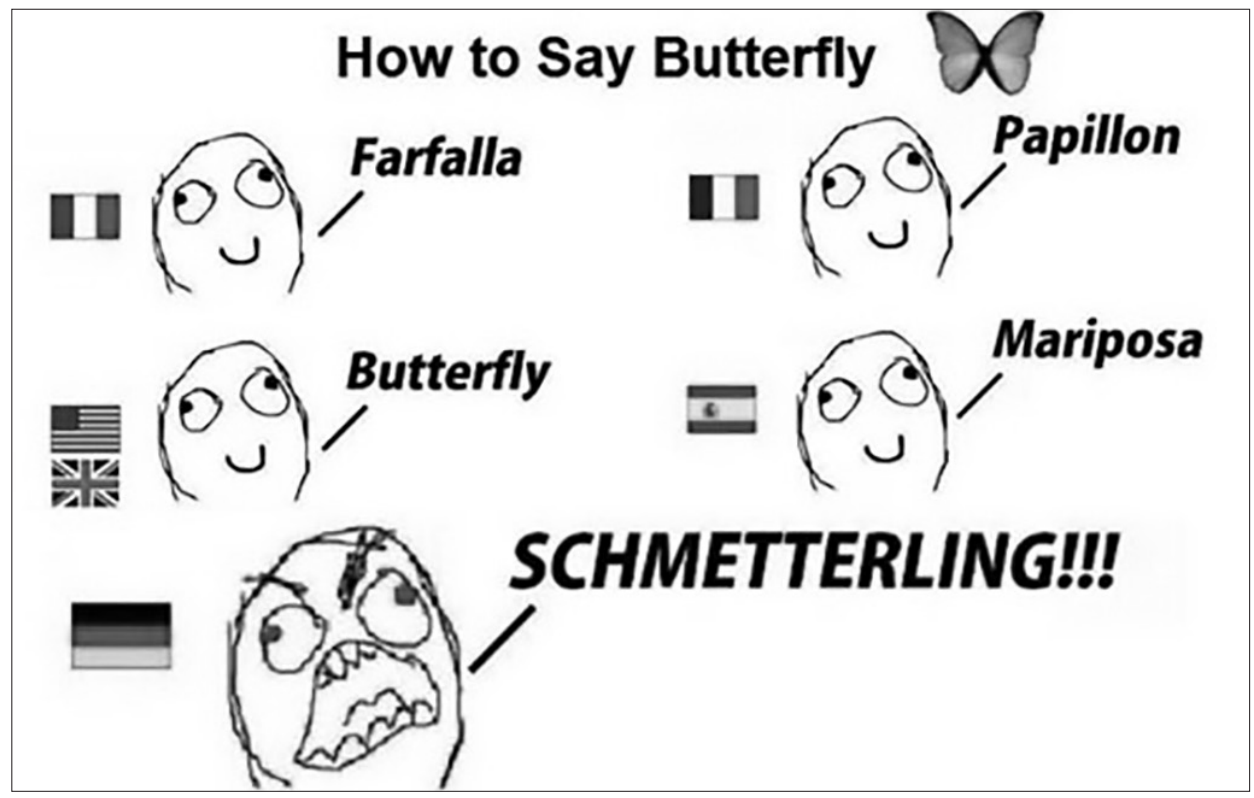

Abbildung 1: How to say butterfly-Meme, https://www.memedroid.com/memes/detail/640043. Zugriff am 17.9.2020.

Deutschen als zu lernende Fremd- bzw. Zweitsprache. Für diese Spracheinstellungen gibt es unzählige Beispiele: in Alltagsgesprächen, im Fernseher, in Zeitungsartikeln, in älteren Schriften, im Internet bzw. in den sozialen Netzwerken (z. B. in der Form sprachvergleichender Memes ${ }^{4}$; Abb. 1-2) usw.

Der Frage nach dem Ursprung von Stereotypen und Spracheinstellungen nähert sich die (sozio)linguistische Forschung hauptsächlich durch zwei Theorien. Die ,inherent value hypothesis“ unterstützt die Idee, dass Sprachen aufgrund inhärenter Eigenschaften als „schön“ oder „hässlich“ empfunden werden. Die "social connotations hypothesis" behauptet hingegen, dass Sprachvarietäten ausschließlich als Folge bestimmter gesellschaftlicher Konnotationen, die sie tragen, als positiv oder negativ empfunden werden (Trudgill/Giles 1976). ${ }^{5}$ Während diese letzte Hypothese mittlerweile deutlich die höchste Akzeptanz genießt, gibt es auch einige (wenige) Ansätze, die das Thema aus einer kognitionswissenschaftlichen Perspektive betrachten. Diese untersuchen, ob es neben den sozialen Konnotationen nicht doch auch etwas Inhärentes gibt (z. B. bestimmte phonetische Eigenschaften), die auf die überwiegend positive oder negative Wahrnehmung mancher Sprachen führen (vgl. Berthele 2010).

\footnotetext{
${ }^{4}$ Humoristische Bilder, die im Netz verbreitet sind.

${ }^{5}$ Quasi dazwischen liegt die Theorie der ,imposed norm hypothesis“ (Giles/Bourhis/Lewis/Trudgill 1974), welche die positive Auswertung mancher Sprachvarietäten mit dem hohen Sozialstatus der Sozialgruppen zusammenbringt, die davon Gebrauch machen (Trudgill/Giles 1976:178).
} 


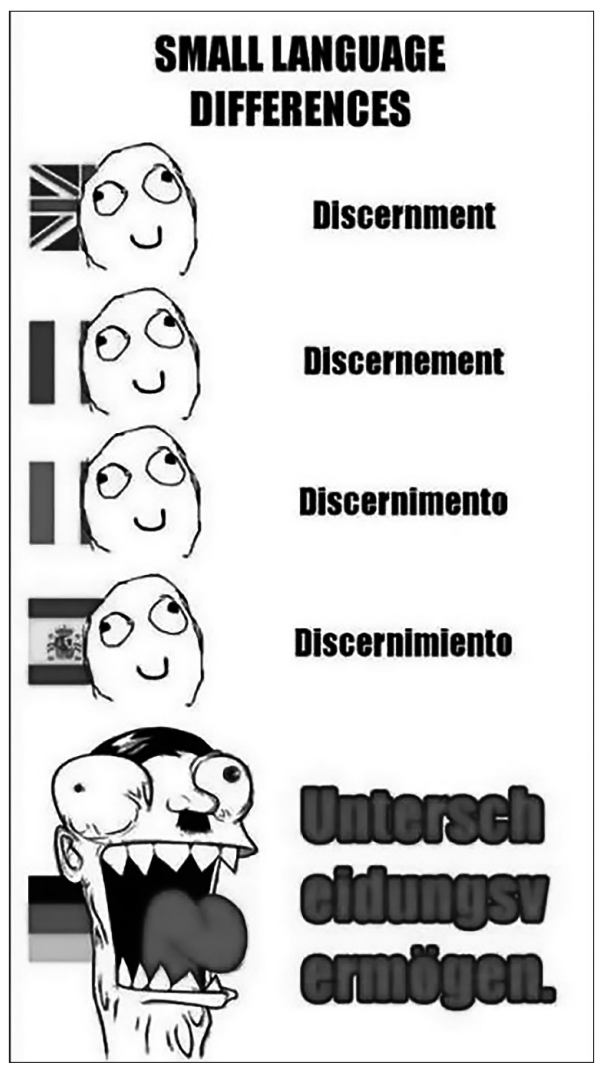

Abbildung 2: Unterscheidungsvermögen-Meme, https://hugelol.com/lol/120122. Zugriff am 17.9.2020.

Wenn die Forschung in diesem Bereich der Sprachwissenschaft allgemein eher lückenhaft ist, fehlen, was das Deutsche betrifft, vor allem systematische bzw. quantitative Daten über die Außenwahrnehmung und -sicht der deutschen Sprache als Ganzes. ${ }^{6}$

\subsection{Fragestellung}

In diesem Rahmen bewegt sich die kommende Analyse, welche einen Beitrag zur Untersuchung der gesellschaftlichen Konnotationen darstellen will, die das Deutsche außerhalb von Deutschland (stereo)typischerweise trägt. Insbesondere wird sich der Beitrag auf folgende Fragen fokussieren:

${ }^{6}$ Für eine Übersicht über die Innensicht vgl. Gärtig/Plewnia/Rothe (2010), für Wahrnehmungsunterschiede zwischen den verschiedenen Varietäten des Deutschen vgl. z. B. Purschke (2011). 
- Welche stereotypischen Meinungen zum Deutschen sind außerhalb von Deutschland besonders gängig? ${ }^{7}$

- Inwiefern finden diese Meinungen über die deutsche Sprache eine Korrespondenz in den Stereotypen, die es über Deutschland und die Deutschen gibt? Auf welche Merkmale der deutschen Kultur können diese Stereotype zurückgeführt werden ${ }^{8}$

- Inwieweit finden manche Stereotype über die deutsche Sprache ihren Ursprung in historischen Ereignissen und Prozessen? ${ }^{9}$

- Wie sehen typische Ausdrücke bzw. Realisierungen dieser Stereotype heutzutage aus? ${ }^{10}$

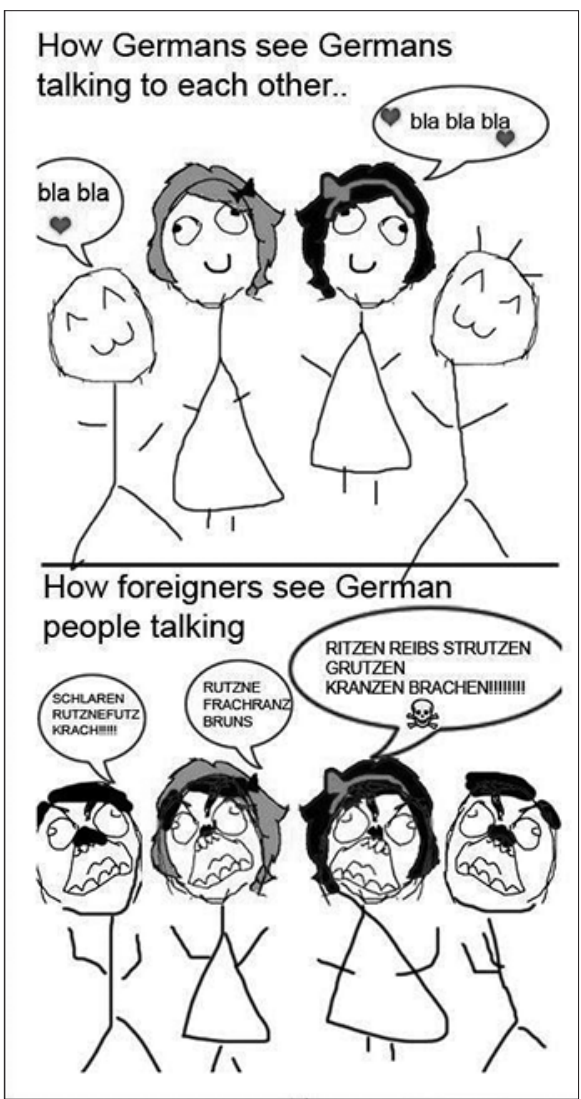

Abbildung 3: How Germans see Germans taking to each other-Meme, https://www.memecenter. com/fun/1791117/how-we-see-german-amp-039-s-talking. Zugriff am 17.9.2020.

7 Wird in Abschnitt 2. behandelt.

8 Wird in Abschnitt 3. behandelt.

9 Wird in den Abschnitten 4.1.-4.3. behandelt.

10 Aktuelle Beispiele sind im ganzen Aufsatz verstreut, werden aber spezifisch in Abschnitt 4.4. behandelt. 
Dabei ist anzumerken, dass diese Analyse keinen Anspruch auf Vollständigkeit erhebt. Es handelt sich vielmehr um eine fragmentarische Rekonstruktion, die qualitativen Charakter aufweist. Was die Sprachurteile vonseiten der Nichtfachleute anbetrifft, gilt dies insbesondere aus dem Grund, dass gesellschaftliche Konnotationen (und alles, was die ästhetische Wahrnehmung von Sprachen sowie allgemein die Spracheinstellungen beeinflusst) aus tiefgehenden Ideen, Gefühlen, Ereignissen und Stimmungen bestehen, welche größtenteils auf unterbewusster Ebene aktiv sind. Somit hat man den Eindruck, man reagiere ausschließlich auf den Klang einer Sprache, während bei der Wahrnehmung eigentlich auch andere versteckte Faktoren eine Rolle spielen. ${ }^{11}$ Aufgrund dieses häufigen (aber auch nicht in jeden Fall zu findenden ${ }^{12}$ ) Mangels einer explizit ausgedrückten Verbindung zwischen Urteilen über die deutsche Sprache und der Geschichte des Landes kann eine derartige Verbindung lediglich hypothetisch angenommen werden und ist methodisch eher schwer (jedoch wahrscheinlich nicht unmöglich) zu fassen. Um aber zu vermeiden, dass dies auf eine reine Spekulation hinausläuft, wird jede Hypothese in der Analyse sorgfältig belegt.

\section{2. (Stereo)typische Meinungen zur deutschen Sprache}

Welche stereotypischen Meinungen zum Deutschen sind außerhalb von Deutschland besonders gängig? Ein guter Ausgangspunkt, um trotz des Mangels an systematischen Daten eine Idee davon zu gewinnen, wie die auf das Deutsche bezogene Außenwahrnehmungslandschaft aussieht, ist die kleine Umfrage, die Gerhard Stickel unter seinen internationalen ${ }^{13}$ Kolleg*innen durchgeführt hat (2003). Die Befragten hatten die Aufgabe bekommen, verbreitete Laienmeinungen über das Deutsche aus ihren jeweiligen Ursprungsländern zu ermitteln. Die Meinungen, die daraus resultieren, könnte man in einem Modell organisieren (Abb. 4). ${ }^{14}$

Dadurch ergibt sich ein vielleicht etwas grober, aber dafür übersichtlicher Eindruck des stereotypischen Empfindens der deutschen Sprache außerhalb von Deutschland. Die Meinungen, die am meisten repräsentiert zu sein scheinen, sind folgende: 1) DIE DEUTSCHE SPRACHE ist schwierig zu lernen; 2) DIE DEUTSCHE SPRACHE zu lernen, ist nützlich; 3) DIE DEUTSCHE SPRACHE

\footnotetext{
11 Wie z. B. eben historische Elemente, die man aber beim Aussprechen einer sprachästhetischen Meinung nur sehr selten als ein Grund, der zu dieser Wahrnehmung beiträgt, anerkennt und nennt.

12 Das Meme in der Abbildung 3 scheint z. B. eine Verbindung zwischen der ästhetischen Fremdwahrnehmung des Deutschen und Deutschlands nationalsozialistischer Vergangenheit aufzuzeigen. 1344 Kolleg*innen aus 15 europäischen und 2 außereuropäischen Ländern: Bosnien, Dänemark, Finnland, Frankreich, Indien, Italien, Japan, Litauen, Norwegen, Polen, Schweden, Schweiz, Spanien, Türkei, Ungarn.

14 In Stickels Erläuterung der Ergebnisse entspricht die Reihenfolge der Antwortschwerpunkte der Reihenfolge der Fragen, die gestellt worden waren.
} 


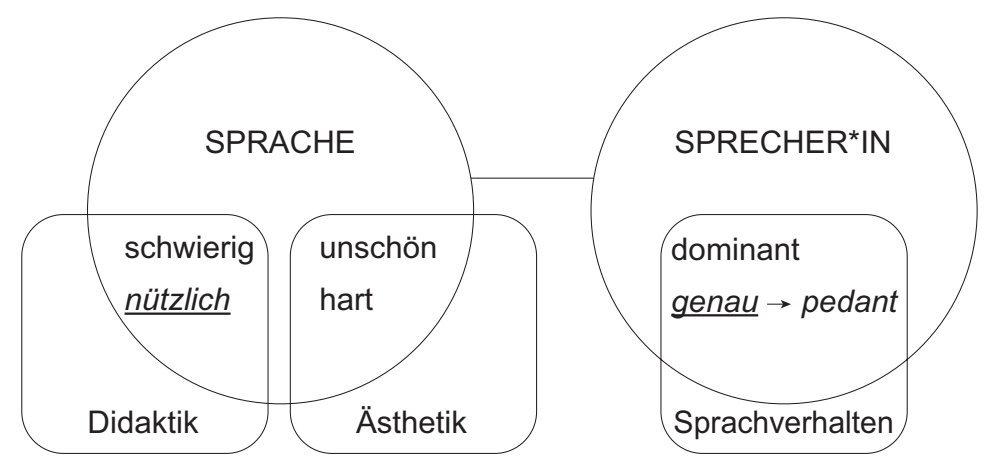

\begin{tabular}{|c|c|c|c|c|}
\hline \multicolumn{5}{|c|}{ LEGENDE } \\
\hline \multicolumn{2}{|c|}{ Schriftart } & \multicolumn{2}{|c|}{ Dimension } & Beantwortet die Frage... \\
\hline \multicolumn{2}{|c|}{ Versalien } & \multicolumn{2}{|r|}{ Objekt (A) } & was/wer? \\
\hline \multirow{2}{*}{ Kursiv } & Unterstrichen & positive & \multirow{2}{*}{ Meinung $(B)$} & \multirow{2}{*}{ wie? } \\
\hline & Nicht unterstrichen & negative & & \\
\hline \multicolumn{2}{|c|}{ Fettdruck } & & Aspekt (C) & wonach? \\
\hline
\end{tabular}

Abbildung 4: Darstellung der Meinungen aus der Stickel-Umfrage.

klingt unschön; 4) DIE DEUTSCHE SPRACHE klingt hart; 5) DIE DEUTSCHEN sprechen mit einem dominanten/aggressiven Ton; 6) die Deutschen argumentieren mit Genauigkeit und Gründlichkeit, was aber schnell auf Pedanterie hinauslaufen kann. Dabei beziehen sich die großgeschriebenen Elemente auf die Dimension (A), z. B. die des Objektes, welche die Frage: „Was/wer (ist das Objekt der Wahrnehmung)?" beantwortet. Objekt sind entweder die Sprache oder die Sprecher*innen. Die kursiv gesetzten sprachlichen Elemente beziehen sich auf die Dimension (B), z. B. die der Meinung, und beantworten somit die Frage: „Wie empfinde ich das Objekt?““. Die Elemente in Fettdruck entsprechen der Dimension (C), die des Aspektes, und beantworten die Frage: „Nach welchem Aspekt wird das Objekt bewertet?"“. Aspekte, die aus den Ergebnissen der Umfrage herausstechen, sind die Didaktik, die Ästhetik (beide auf Sprache bezogen) und das Sprachverhalten (auf die Sprecher*innen bezogen).

Die Nützlichkeit (2) der Sprache wird vor allem in Bezug auf die Wirtschaft genannt und zum Teil auch noch auf die Wissenschaft/Philosophie. Die empfundene Härte (4) des Deutschen wird typischerweise als negativ wahrgenommen und führt direkt zur Bezeichnung der Sprache als unschön (3). ${ }^{15}$ Diese Wahrneh-

15 „[W]obei der Schönheitsmangel oder gar die Hässlichkeit vor allem mit phonischen Eigenschaften begründet wird“; „Konsonantenreichtum, die vielen s-Laute, die Achs und Ichs“ (Stickel 2003:6). 
mung wird durch verschiedene Adjektive bzw. Formulierungen ausgedrückt. ${ }^{16}$ Darunter erinnert die Aussage, dass das Deutsche eine Sprache sei, die ,gebellt“ wird (Stickel 2003:6), an das Zitat von Primo Levi, mit dem dieser Aufsatz begonnen hat. Zum Eindruck der Dominanz (5) gehört u. a. die mehrmals vorkommende Anmerkung, dass Deutsche eine „Tendenz zum lautstarken Reden“ haben. Der laute Ton wird u. a. als „,̈̈berheblich“, „herrisch“, ,,aggressiv“, ,,autoritär“ empfunden. Dabei ist es interessant, anzumerken, dass dasselbe Stereotyp oft auch für Südländer*innen gilt, und dass das Empfinden in diesem Fall aber sehr anders ist: Das laute Sprechen gilt bei Südländer*innen zwar oft als nervig, aber auch als ein Zeichen der Offenheit und Wärme. Insgesamt sind die im Ausland verbreitetsten Meinungen, die diese ,informelle“ Befragung sammeln konnte, eher negativ.

\section{Stereotype und Kulturstandards}

Obwohl Stereotype ein komplexes soziologisches Phänomen darstellen, wird die folgende Definition für unsere Zwecke ausreichen:

(a) stereotypes are aids to explanation, (b) stereotypes are energy-saving devices, and (c) stereotypes are shared group beliefs. The first of these implies that stereotypes should form so as to help the perceiver make sense of a situation, the second implies that stereotypes should form to reduce effort on the part of the perceiver, and the third implies that stereotypes should be formed in line with the accepted views or norms of social groups that the perceiver belongs to (McGarty/Yzerbyt/Spears 2002:2).

Stereotype entstehen durch einen kontrastiven Prozess: Sie sind meistens die Folge der Fremdwahrnehmung eines Verhaltens, welches in einer bestimmten Kultur vielleicht „unmarkiert“ ist, aber in anderen eher „,markiert" ist. Um sich das „fremde“ Verhalten zu erklären (a) und mit diesem Verhalten auch in der Zukunft praktischer umgehen zu können (b), wird dessen Interpretation oft vereinfacht. Dieses praxisorientierte Wissen über eine bestimmte Kultur (es handelt sich nämlich in diesem Fall nicht um ein Wissen, das darauf zielt, eine Kultur tatsächlich kennen zu lernen) wird unter Individuen anderer Kulturen verbreitet (c). Der folgende Auszug aus „Gödel, Escher, Bach“ (Hofstadter ${ }^{20} 1999$ ) erklärt diesbezüglich, wie unser Verständnis eines Elements ein zunehmendes, annäherndes Verfahren darstellt, bei dem sich Menschen anfangs größtenteils des ihnen verfügbaren Wissens über die „Klasse“ bedienen, zu der das einzelne Element gehört,

16 Z. B. „hässlich“, ,trocken“, ,kalt““; ,unmelodisch“, „,herrisch“, „,militärisch“ „,eckig“ usw. (Stikkel 2003:6). 
und erst in einem zweiten Moment dank des Erwerbes neuer Informationen über das Element ihre Wahrnehmung davon allmählich neu ausrichten und präzisieren:

[...] instance symbols often inherit many of their properties from the classes to which those instances belong. If I tell you I went to see a movie, you will begin „minting“ a fresh new instance symbol for that particular movie; but in absence of more information, the new instance symbol will have to lean rather heavily on your pre-existing class symbol for „movie“. Unconsciously, you will rely on a host of presuppositions about that movie [...]. These are built into the class symbol as expected links to other symbols (i.e. potential triggering relations), and are called „default options“. In any freshly minted instance symbol, the default options can easily be overridden, but unless this is explicitly done, they will remain in the instance symbol, inherited from its class symbol. Until they are overridden, they provide some preliminary basis for you to think about the new instance-for example, the movie I went to see-by using the reasonable guesses which are supplied by the „stereotype“, or class symbol (Hofstadter ${ }^{20} 1999: 352-353$; Formatierung von d. Verf. adaptiert).

Dieses handelsorientierte Vereinfachen ist beim Kontakt mit anderen Kulturen allerdings nicht notwendigerweise negativ, solange dabei das Bewusstsein besteht, dass es sich eben um eine Vereinfachung handelt: „Zu sagen, die Typisierungen gleiten zu leicht ins Negative ab, provozieren fast automatisch feindselige Haltungen, und deshalb sollte man sie tunlichst unterlassen, ist zwar gut gemeint, aber naiv. Typisierungen sind immer ein wichtiges Instrument der Erkenntnis und der Orientierung, und das paradoxerweise umso mehr, je komplexer die Wirklichkeit ist [...]" (Schroll-Machl 2002:22).

Es muss außerdem berücksichtigt werden, dass Stereotype meistens auf tatsächlich bestehenden Kulturstandards ${ }^{17}$ basieren und als deren versteiftes Verständnis sie betrachtet werden können. Eine scharfsinnige Übersicht über deutsche Kulturstandards bietet Schroll-Machl (2002) ${ }^{18}$, wo folgende Kulturstandards Deutschlands identifiziert werden: (1) Sachorientierung; (2) Wertschätzung von Strukturen und Regeln; (3) regelorientierte, internalisierte Kontrolle; (4) Zeitplanung; (5) Trennung von Persönlichkeits- und Lebensbereichen; (6) Direktheit der Kommunikation.

Inwiefern finden die im Abschnitt 2. identifizierten Meinungen über die deutsche Sprache eine Korrespondenz in diesen Kulturstandards? Wenn man die Elemente dieser beiden Kategorien intuitiv miteinander verbinden möchte (Abb. 5), scheint die Korrespondenz zwischen den Kulturstandards und den Meinungen, die

17 Mehr über das Konzept von Kulturstandards vgl. Thomas (1999).

18 Es geht in dem Beitrag ausdrücklich um das Geschäftsumfeld, allerdings eignen sich die identifizierten Kulturstandards auch für eine allgemeinere Anwendung sehr gut. 


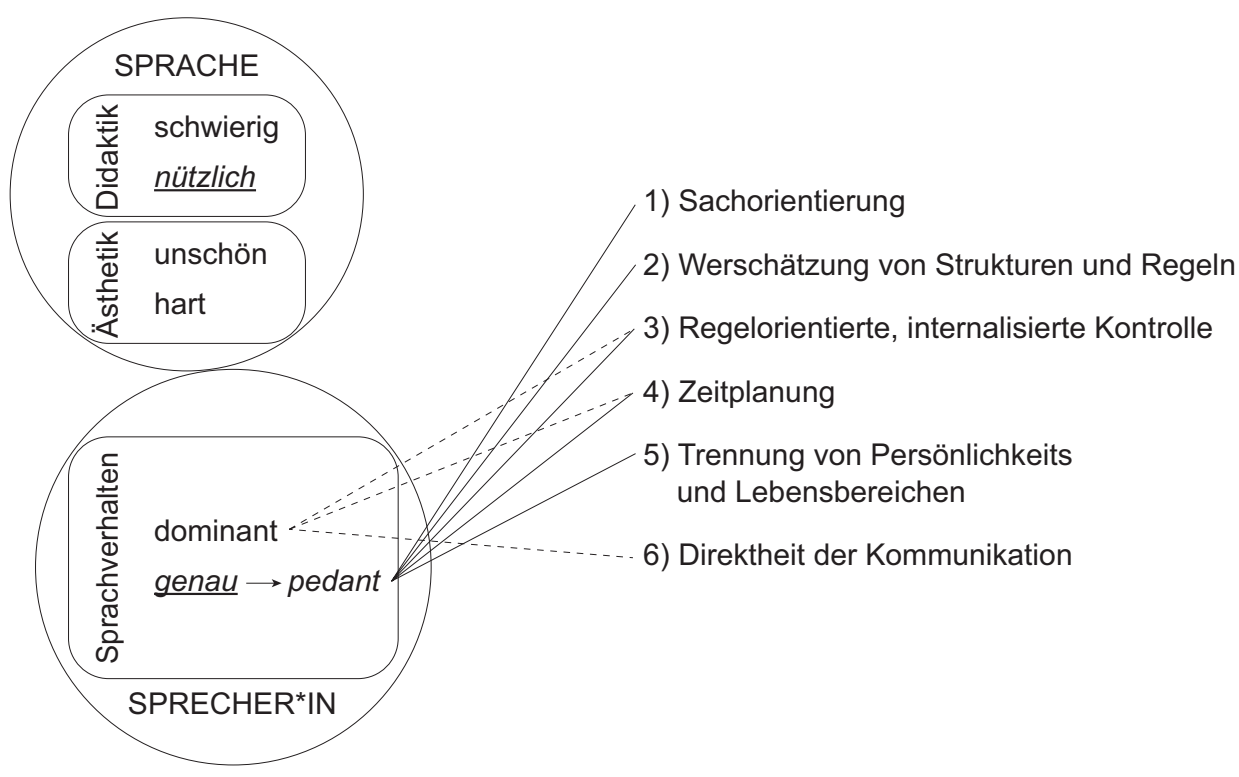

Abbildung 5: Darstellung der Korrespondenz zwischen Meinungen aus der Stickel-Umfrage und deutschen Kulturstandards.

sich auf die Sprecher*innen (also auf die Deutschen) beziehen, tatsächlich sehr plausibel. Anders sieht es bei den rein sprachbezogenen Meinungen aus, wo eine direkte Verbindung mit den Kulturstandards als weniger sinnvoll erscheint. Es wurden im Abschnitt 2 allerdings auch einige Adjektive zitiert, die in den Antworten auf Stickels Frage zum Sprachverhalten der Deutschen erschienen sind, darunter z. B. „aggressiv", welches sich auch zum Beschreiben des Klanges einer Sprache eignen würde. In vergleichbarer Weise passen wiederum Adjektive wie „herrisch“ und „militärisch“, die in den Antworten direkt auf die deutsche Sprache bezogen waren, perfekt in die Idee der Dominanz. Es handelt sich bei „Härte“ und „Dominanz“ also um Begriffe, die in diesem Kontext konzeptuell sehr nah beieinanderstehen. Dazu passend teilen eigentlich alle Kulturstandards von Schroll-Machl die Gemeinsamkeit, dass sie in ihrer negativeren Deutung einen gewissen Eindruck von Härte und Strenge vermitteln. Dies scheint die Aussage zu rechtfertigen, dass das Stereotyp der Unangenehmheit der deutschen Sprache sich wahrscheinlich in diese generelle Wahrnehmung der deutschen Härte eingliedert. 


\section{Was historisch und gesellschaftlich wohl dahintersteckt: fragmentarisch vom sechzehnten Jahrhundert bis zur heutigen Zeit}

\subsection{Lügen, Pferde und Kriege: auf Deutsch gesagt}

Inwieweit finden manche Stereotype über die deutsche Sprache ihren Ursprung in historischen Ereignissen und Prozessen? Um diese Frage zu beantworten, wird im Folgenden eine Reihe von typischen Ausdrücken bzw. Realisierungen der Stereotype über das Deutsche untersucht, die in der Literatur auffindbar sind. Parallel werden, wo das möglich ist, die entsprechenden Zusammenhänge geklärt.

„Im Deutschen lügt man, wenn man höflich ist", antwortet der Baccalaureus in Goethes Faust, als ihn Mephisto beschuldigt, grob zu sein (von Goethe 2005:514). Dieses Zitat wird heute noch in Bezug auf ein bestimmtes Verhalten oder eine bestimmte Äußerung verwendet, deren Höflichkeit/Zuvorkommenheit man als künstliche Schmeichelei auffasst. Auch die Redensart „auf Deutsch gesagt" oder „mit jemandem Deutsch reden“ bezeichnet umgangssprachlich einen direkten Kommunikationsstil, wo eine Wahrheit/Meinung ungeschminkt ausgedrückt wird. Eine weitere Bedeutungsnuance, welche diese Redewendung tragen kann, ist die eines verständlichen oder volkstümlichen Redens, z. B. im 16. Jahrhundert etwa im Vergleich zur Verwendung des Lateinischen (der Gelehrtensprache). Die Verwendung dieses Ausdruckes sowie die des Zitates von Goethe sind ein gutes Beispiel dafür, wie der deutschen Sprache üblicherweise bestimmte Eigenschaften zugeordnet werden: Das Deutsche ist direkt, klar, aufrichtig, unverblümt, und diese Direktheit kann als hart und unhöflich rüberkommen.

Das 16. Jahrhundert, wo die oben erwähnte Redensart möglicherweise ihren Ursprung hat, ist auch die Zeit von Karl V., dem (teilweise irrtümlich) folgender Satz zugeschrieben wird: ,Wenn ich mich im Gebet an Gott wende, dann auf Spanisch; mit meiner Geliebten spreche ich Italienisch, mit meinen Freunden Französisch; mit meinen Pferden spreche ich Deutsch“. Die erste bekannte Fassung dieser Anekdote weist zwei unterschiedliche Formulierungen auf, die aber beide etwas von diesem Bild der Härte des Deutschen wiedergeben. Die erste Version beschreibt die deutsche Sprache als eine Militärsprache; die zweite beschreibt sie als eine Sprache, die sich zum Drohen und Befehlen besonders gut eignet (Fabrici 1601:64). Diese Anekdote wurde im Laufe der Jahre in verschiedenen Texten wiedergegeben, übernommen und verdreht. Darüber wird z. B. 1963 in der Glosse von Josef Müller-Marein mit dem Titel „Das Pferde-Plagiat“ und 1966 im Zeitungsartikel „Wie sprach Kaiser Karl mit seinem Pferd?“ von dem deutschen Historiker Arno Borst berichtet. Die Glosse zitiert noch dazu Friedrich den Großen („Das Deutsche ist ein barbarischer Jargon, gerade noch geeignet, um mit seinen Pferden zu sprechen“; zit. n. Müller-Marein 1963) und einen von Voltaire 
geschriebenen Brief aus Potsdam: „Hier spricht man nur unsere Sprache. Das Deutsche ist bloß für Soldaten und Pferde“ (zit. n. Müller-Marein 1963). Bemerkenswert ist bei Karl V. aber noch eine Aussage, die das große Thema der von den Personen der Bibel im Paradies gesprochenen Sprache einführt ${ }^{19}$ : Karl V. habe behauptet, dass Gott bestimmt Deutsch gesprochen haben muss, als er Adam und Eva aus dem Paradies vertrieb, ,denn das ist eine blitzende und donnernde Sprache“" (vgl. Borst 1966:1). Auch der französische Autor Dominique Bouhours teilte mit seinen Lesern seine Meinung über mehrere Sprachen. Das Deutsche definiert er dabei als rau und grob (Bouhours 1708:67; zit. n. Jones 1999:120). Weiter geht es mit dem dänischen Dichter Ludvig Holberg, der am Anfang des 18. Jahrhunderts berichtet, ein französisches Mädchen habe ihm bei seinem Pariser Besuch mitgeteilt, ,er spreche Französisch wie ein deutsches Pferd. Im Munde der Magd wurde der letzte Rest des kaiserlichen Ausspruchs verdreht" (Borst 1966:4). Es handelte sich dabei aber noch lange nicht um den letzten Rest dieses Ausspruchs: Jonathan Swift beschreibt in seinem „Gulliver's Travels“ aus dem 18. Jahrhundert das Land der Pferde: „In speaking, they pronounced through the nose and throat, and their language approaches nearest to the High-Dutch, or German, of any I know in Europe; but is much more graceful and significant. The emperor Charles V. made almost the same observation, when he said, that if he were to speak to his horse, it should be in High-Dutch"“ (Swift 1999:182; Formatierung von d. Verf. adaptiert). Der letzte von Borst erwähnte Autor ${ }^{20}$ ist der Österreicher Franz Grillparzer, der 1845 auch seine Meinung zum Thema Europäische Sprachen und deren passendste Funktion ausdrückte und Deutsch als eine der Bildungssprachen (zusammen mit Griechisch und Lateinisch) und als die Muttersprache des Dichters, die „zum Sagen“ (Borst 1966:5) geeignet ist, bezeichnete. Das erinnert an die Meinungen (2) und (6) aus dem Abschnitt 2: Die deutsche Sprache ist nützlich (etwa als Sprache der Wissenschaft und Philosophie) und genau/gründlich/klar.

Auch heute noch ist ein Zitat über die deutsche Sprache besonders beliebt: „Life is too short to learn German“. Gesagt soll das Richard Porson haben, Belege hierfür findet man in Peacock (1861:21) und Froude/Tulloch/Carlyle $(1860: 455)^{21}$. Bei dieser etwas ironischen Aussage wird diesmal die Schwierigkeit der deutschen Sprache für die Lernenden, das im Abschnitt 2 identifizierte Stereotyp (1), aufgenommen. Im ähnlichen Stil wie das angebliche Zitat von Porson ist der oft zitierte Beitrag des amerikanischen Schriftstellers Mark Twain, der 1880 in seinem humoristisch-satirischen Essay „The Awful German Language“ (2016) seine Ansichten bezüglich der deutschen Sprache aus der Perspektive eines

19 Für weitere Beiträge über dieses Thema vgl. Jones (1999), Delumeau (2000).

20 Weitere historische Figuren, die auf die Worte Karls V. Bezug nahmen, sind laut Borst Graf Chesterfield 1762, der russische Gelehrte Lomonossow 1755 und August Willhelm Schlegel 1798 (Borst 1966:5).

21 Das Zitat wird im Netz oft auch anderen Autoren zugeordnet, wie z. B. Oscar Wilde - nur was Porson betrifft, konnte ich allerdings Belege finden (wenn auch nur indirekte). 
Lerners englischer Muttersprache präsentiert - und somit aus dem didaktischen Aspekt (C) unseres Modells (Abb. 4). Dabei konzentriert sich Twain vor allem auf morphologische, syntaktische und lexikalische Aspekte, wobei er am Ende der kurzen Abhandlung noch auf phonologische Eigenschaften eingeht.

\subsection{Dichter und Denker, Richter und Henker}

Bisher waren das eher negative Ansichten über die deutsche Sprache, die vorgestellt wurden. Das Deutsche sowie die Deutschen genossen aber in manchen Phasen ihrer Geschichte einen sehr guten Ruf. So findet man z. B. in „Ernest Maltravers or the Eleusinia“" von Edward Bulwer (1866) folgende Widmung: „To the great German people, a race of thinkers and of critics". Dieser Satz wurde als ein möglicher Ursprung für die - auf das deutsche Volk bezogen - entstandene Bezeichnung „Dichter und Denker“ gesehen (Kainz 1974:338). Vielleicht am meisten verbreitet ist aber die Idee, dass der Ausdruck ursprünglich aus den „Volksmärchen der Deutschen“ (zwischen 1782 und 1786 herausgegeben) von Johann Karl August Musäus stammt: „Was wäre das enthusiastische Volk unserer Denker, Dichter, Schweber, Seher ohne die glücklichen Einflüsse der Fantasie?“ (zit. n. Depenheuer/Peifer 2008:47). ${ }^{22}$ Rainer Traub (2007) schreibt: „Kaum eine Vorstellung hat dem Selbstbild der Deutschen und dem Fremdbild, das sich andere von ihnen machten, so zäh und folgenreich angehaftet wie die vom, Volk der Dichter und Denker“". Dementsprechend galt das Deutsche in dieser Zeit als die Sprache der Dichter und Denker. Aber genauso wie das Stereotyp der Genauigkeit/Gründlichkeit/Klarheit der deutschen Sprache in ihrer negativeren Deutung zur Pedanterie wird (vgl. Abschnitt 2), kann auch diese Vorstellung abwertende Deutungen tragen:

Die Deutschen thun nicht viel, aber sie schreiben desto mehr. Wenn dereinst ein Bürger der kommenden Jahrhunderte auf den gegenwärtigen Zeitpunkt der deutschen Geschichte zurückblickt, so werden ihm mehr Bücher als Menschen vorkommen. [...] Er wird sagen, wir haben geschlafen und in Büchern geträumt. [...] Das sinnige deutsche Volk liebt es zu denken und zu dichten, und zum Schreiben hat es immer Zeit (Menzel ${ }^{2}$ 1836:3).

Dichter und Denker, vor allem Dichter, gab es auch anderswo, hatte es vor allem anderswo in größerer Zahl und Regelmäßigkeit gegeben. Wenn dieses Wort also einen Sinn haben sollte, mußte es etwas anderes bedeuten, mußte in ihm eine Einschränkung, eine Art Selbstkritik enthalten sein. Es konnte

22 Die Wendung bezieht sich insbesondere auf das deutsche intellektuelle Umfeld des achtzehnten und des neunzehnten Jahrhunderts und somit auf die große Zeit der deutschen Klassik und Romantik: die Zeit von (unter anderen) Lessing, den Schlegels, Goethe, Schiller, Hegel, Fichte und Kant. 
dann nur meinen, daß die deutschen Dichter und Denker der höchste Ruhm der Deutschen waren, vielleicht sogar ihr einziger, worin sie sich denn von Franzosen oder Spaniern oder Engländern unterschieden hätten. Und so wurde es aufgefaßt, so kennzeichnete es gleichzeitig Genügen und Ungenügen der Deutschen an sich selbst (Boehlich 1964).

Der Titel des Volkes der „Dichter und Denker“ verbreitete sich besonders dank Mme Germaine de Staël bis in den ganzen Mittelmeerraum (vgl. Milling 2010:88). In ihrem bekannten Werk „De l'Allemagne“ beschreibt de Staël ihre Eindrücke über Deutschland und über die deutschen Gelehrten. ${ }^{23}$ Der ,selbstgewählte Ehrentitel“ (Boehlich 1964) sei möglicherweise auch ironisch gemeint, „,ein Beispiel von Selbstironie eines als humorlos geltenden Volkes“" (Menasse 2004). Er scheint außerdem auch eine Art Stolz auszudrücken. In einer instabilen Zeit, als Deutschland politisch gesehen noch kein Land war, habe diese „,nicht nur für Deutschland auffällige Blütezeit“ den Deutschen einen „neuen Nationalstolz“ geschenkt (Boehlich 1964). Diese neue bzw. neugeborene Wertschätzung des Deutschen führt z. B. Herder dazu, eine Akademie für die „Reinigkeit“ der Sprache vorzuschlagen (Herder 1803:227). In einem Gedichtfragment mit dem Titel „Deutsche Größe" drückt Schiller 1902 sogar die Meinung aus, dass die deutsche Sprache die Welt beherrsche. Unabhängig davon, ob dieser Titel ironischen oder eher selbstironischen Charakter hatte, oder ob er doch mit aller Ernsthaftigkeit benutzt wurde - und wenn, ob mit positiver oder mit negativer Deutung, zweifellos genossen die deutsche Sprache und Kultur zu dieser Zeit eine ausgeprägte und weit verbreitete Hochachtung. Dass die deutsche Sprache unangenehm klinge, scheint die Wahrnehmung zu dieser Zeit nicht mehr zu prägen.

Heinrich Heine schreibt dann jedoch: „Der Patriotismus des Deutschen [...] besteht darin, dass sein Herz enger wird, dass es sich zusammenzieht wie Leder in der Kälte, dass er das Fremdländische hasst, dass er nicht mehr Weltbürger, nicht mehr Europäer, sondern nur ein enger Deutscher sein will““ (Heine 1876:51f.). Dementsprechend zeichnet sich bald ein Bruch ab: „Der selbstgewählte Ehrentitel sagte ihnen [den Deutschen] zu und sagte ihnen doch wieder nicht zu. Sie wollten nicht länger nur dichten und denken oder träumen (was bald denselben Sinn erhielt); sie wurden tatendurstig. [...] Sie schrieben ihre Literatur ab [...] und wandten sich der Politik zu“" (Boehlich 1964).

Was diesem Wandel folgt, ist, dass aus dem Volk der Dichter und Denker ein „Volk der Richter und Henker“ wird (Kraus 1967:29). „Sprachen haben ja, so wie jede andere menschliche, gesellschaftliche oder nationale Lebensäußerung, ein Image“ (Menasse 2004): Mit dem deutsch-französischen Krieg von 1870, dem

23 Vgl. de Staël-Holstein (1815:131f.). 
Ersten und vor allem dem Zweiten Weltkrieg veränderte sich das Image der deutschen Sprache und das der Deutschen zweifellos ganz grundlegend, mit Folgen, die bis heute deutlich spürbar sind. Die früher von der Epoche der „Dichter und Denker" aufgerufenen Vorstellungen hatten sich mit den Kriegen aufgelöst:

[...] wenig hat dem Dichten in deutscher Sprache in neuerer Zeit so nachhaltigen Schaden zugefügt wie die Restaurationsanstrengungen des ,Dichterund-Denker'-Selbstbildes nach 1945. Nach der Nazi-Zeit war alles kaputt, was Deutschland zur Ausstattung von Selbstgefühl positiv ins Treffen führen konnte oder je wollte. Politisch kriminell geworden, wirtschaftlich am Boden und von der Hilfe der Siegermächte abhängig, ideologisch verwirrt und teils willfährig teils verhohlen aggressiv im Zustand der Umschulung, geschlagen befreit (Menasse 2004).

Wie zeigt sich das konkret? Einen interessanten Beleg dafür stellt der Text Gian Enrico Rusconis dar, der noch 2016 auf der italienischen Webseite des GoehteInstituts - unter dem „Über-uns“-Reiter zu lesen war:

In der unmittelbaren Nachkriegszeit [...] stellte das Ziel, ein neues gegenseitiges Verständnis zwischen den Gesellschaften und Kulturen Italiens und Deutschlands herzustellen, die Goethe-Institute bei ihrer Gründung in den fünfziger Jahren in Italien vor drei Aufgaben: die vor allem in der letzten Phase des Krieges entstandene gegenseitige Feindseligkeit, die Vorurteile und die Klischeebilder abzubauen, die der Wiederaufnahme der Kontakte zwischen den beiden Völkern und den beiden Kulturen im Wege standen; die Kenntnisse der deutschen Kultur in Italien über den Kreis der Spezialisten hinaus stetig zu verbessern; eine enge Zusammenarbeit zwischen deutschen und italienischen Instituten und Persönlichkeiten herzustellen. Grundlage dieser Aktivitäten war selbstverständlich die Förderung und Verbreitung der deutschen Sprache (Rusconi 2001a:49).

Dieser Auszug bringt deutlich zur Geltung, welch große Anstrengung nötig war und teilweise immer noch ist, sich der Erbschaft des Zweiten Weltkriegs zu stellen und die daraus resultierenden Vorurteile sowie mit Deutschland verbundenen negativen Vorstellungen zu bekämpfen. Während sich die dritte zitierte Aufgabe des Instituts auf das große Thema „Europa“ bezieht, ist es noch anzumerken, dass in der italienischen Version des Textes die zweite Aufgabe dahingehend präzisiert wird, dass unter „deutscher Kultur“ nicht nur die „klassische“ zu verstehen ist, sondern auch - und vor allem - die „neue“ deutsche Kultur. ${ }^{24}$ Dieses Konzept

24 ,[F]ar conoscere sempre meglio in Italia, al di là della cerchia degli specialisti, la cultura tedesca - non soltanto quella ,classica“ ma anche e soprattutto quella ,nuova““ (Rusconi 2001b; Formatierung von d. Verf. adaptiert). 
wird sich im Rahmen der kommenden Analyse der Einflüsse des Ersten und des Zweiten Weltkrieges auf die Fremdwahrnehmung der Deutschen und des Deutschen als wesentlich erweisen.

\subsection{Von den Weltkriegen bis zur Europäischen Union}

Ein vielsagendes Bespiel der „Dämonisierung“ der deutschen Sprache nach dem Ausbruch des Ersten Weltkrieges stellt der Fall des Status des Deutschen in den USA dar. Vor dem Ersten Weltkrieg war die Gemeinschaft der deutschen Einwanderer ein bedeutungsvoller Teil der amerikanischen Gesellschaft, so dass zwischen dem Ende des 19. und dem Anfang des 20. Jahrhunderts Deutsch die meist unterrichtete Fremdsprache in den USA war (Handschin 1913; Zeydel 1964). Die positive Einstellung der deutschen Sprache gegenüber fand sich auch in offiziellen Äußerungen, so heißt es z. B. 1870 beim Commissioner of Education: „the German language has actually become the second language of our Republic, and a knowledge of German is now considered essential to a finished education" (zit. n. Zeydel 1964:345).

Mit dem Ausbruch des Ersten Weltkrieges entsteht aber ein „anti-German sentiment" (Pavlenko 2003:318), das sich im Verlauf des Krieges allmählich und kontinuierlich steigert, bis im April 1917 die Kriegserklärung der Vereinigten Staaten an Deutschland zu einer Eskalation der ,anti-German campaign“ führt. Diese Kampagne war ein genereller Angriff auf die Nation einschließlich ihrer Sprache und Kultur, und sie zielte auch auf die deutsch-amerikanische Gemeinschaft. Nun erschienen zahlreiche Artikel gegen das Lernen der deutschen Sprache als der Sprache des Feindes. Die Schulbehörden erhielten Petitionen, den Deutschunterricht abzubrechen, und Sprecher*innen des Deutschen wurden pauschal beschuldigt, an subversiven pro-deutschen Aktivitäten teilgenommen zu haben. In einzelnen Gemeinden waren Deutsch-Amerikaner Drohungen, Einschüchterungen und Prügeln ausgesetzt. Deutsche Bücher wurden aus den Kirchen-, Schul- und Universitätsbibliotheken entfernt und zerstört, oder sogar öffentlich verbrannt. Mehrere deutsche Professor*innen an Hochschuleinrichtungen wurden wegen mangelnder Unterstützung für die USA und ihre Verbündeten entlassen, und an der Universität von Michigan wurde der größte Teil des deutschen Programms abgebaut (Luebke 1980, Wiley 1998, Pavlenko 2003). Zwischen 1917 und 1922 „German as a foreign language was practically legislated out of existence“ (Pavlenko 2003:318) - wobei diese Politik dann häufig auch weitere Fremdsprachen betraf. So erließen z. B. die Staaten Indiana, Louisiana und Ohio Regulierungen, durch die das Unterrichten der deutschen Sprache, welche „als ,Hunnensprache " gebrandmarkt" worden war (Brunner 2014), für illegal erklärt wird (vgl. Pavlenko 2003:318). Der „Widerstand gegen alles Deutsche“, nimmt „hysterische Züge an“: Unter anderem werden auch Straßen „flugs umbenannt, 
aus der ,Deutschen Sparkasse' wurde die ,Central Savings Bank', die ,Germania Life Insurance Co.' wurde zur ,Guardian Life Insurance Co.'. Das Sauerkraut, bis dahin allgemein unter diesem Wort bekannt, hieß fortan liberty cabbage, und aus dem Hamburger wurde das Salisbury Steak" (Formatierung von d. Verf. adaptiert). Im April 1918 erklärt die New York Times die deutsche Sprache ,schlicht für tot" (Brunner 2014). Dabei gab es allerdings auch Stimmen, die sich im Rahmen der damals aktuellen Debatte über die Wichtigkeit des Fremdsprachenlernens gegen die deutschenfeindliche Kampagne wendeten. Pavlenko (2003) weist auf drei verschiedene, sich in diese Debatte verflechtende Diskurse hin, von denen zwei an dieser Stelle besonders relevant erscheinen. In einem ersten Diskurs wird die Sprache als ,major influence on the thought of its speakers“ betrachtet (Pavlenko 2003:319); er stützte sich ironischerweise gerade auf Theorien, die von deutschen und deutschamerikanischen Wissenschaftlern bzw. Philosophen worden waren (z. B. W. v. Humboldt, Boas, Sapir und Whorf). Sprachen seien „not equal in theoretical, practical, and moral values“, junge Amerikaner müssten daher vor Sprachen beschützt werden, von denen sie hätten „verseucht“ werden können. „German in particular was at fault as it was the language of a nation that had ,lost all moral sense “25“ (Pavlenko 2003:319). Diese explizit ausgedrückte Verbindung zwischen den Untaten und Verbrechen der Deutschen und ihrer Sprache fand zahlreiche Wortführer. In der „Educational Review“ erklärte z. B. Gordy, dass die Sprache des „Teutonismus“, „[which] prides itself in its inhumanity for it murders innocent children, rapes women, and mutilates the bodies of innocent men" (1918:262), auf keinen Fall in die amerikanische Bildung passe. Ähnliche Beiträge, wie dass eine Sprache ,which produces a people of ruthless conquestadors [sic!] such as now exists in Germany, is not a fit language to teach clean and pure American boys and girls“ (zit. n. Luebke 1980:5; Formatierung von d. Verf. adaptiert), sind in den Prospektmaterialien der „American Defense League“ zu finden. Der zweite, mit dem ersten eng verbundene, Diskurs „eschewed a direct critique of language, focusing instead on the practices and behaviours of its speakers, in particular German-born language instructors who presumably at times

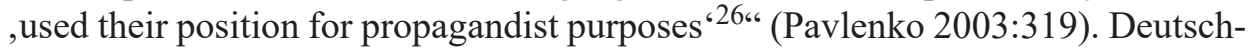
landfreundliche Mitglieder*innen der amerikanischen Gesellschaft planten laut dem Dean des College of Education der Universität Minnesota, ihre Ziele „by having German teachers teaching German ideals through the German language“ zu erreichen (zit. n. Luebke 1980:5). Obwohl die Satzung des „English-only movement" wegen ihrer Verfassungsfeindlichkeit nicht aufrechterhalten werden konnte,

25 Barnes (1918:188).

${ }^{26}$ Fitz-Gerald (1918:59). 
the damage to foreign-language education - and in particular to German study - was already done. While in 1917 there were 31,000 students taking German in Milwaukee alone, there were none two years later. By 1922, high schools all over the country had less than 14,000 students of German, $0.5 \%$ of the overall high school enrolment (as compared to 15.5\% in French and 10\% in Spanish) (Pavlenko 2003:321).

Ganz anders wurde das Thema des Fremdsprachenunterrichts zu dieser Zeit in der Ex-Sowjetunion behandelt, da ab den 20er Jahren die „Sprache des Feindes“ und ihre Beherrschung als ein sehr mächtiges Instrument für die ideologische Propaganda des Sozialismus und des Kommunismus betrachtet wurde. Selbst als im Zweiten Weltkrieg mit dem Kriegseintritt im Juni 1941 auch in der Sowjetunion eine aggressive deutschenfeindlichen Kampagne ausbricht, wurde das Unterrichten des Deutschen als Fremdsprache nicht so dämonisiert wie in den USA, ,as the authorities realised that it represented an important linguistic resource [...] for the purposes of national security and economic and technological developments“ (Pavlenko 2003:322). Allerdings erstellte ,the educational establishment [...] teaching materials and curricula permeated by texts, vocabulary and exercises of ideological value“ (Pavlenko 2003:323), um einer möglichen Verseuchung der Kinder durch die von ihnen gelernten Sprachen vorzubeugen.

Einen weiteren repräsentativen Fall stellt die britische Wahrnehmung von Deutschland und den Deutschen nach 1945 und die Stereotypisierung der Deutschen in der britischen Presse dar:

Although Anglo-German relations since 1945 have by and large been friendly at the level of the political elite, on a wider scale British perceptions of Germany and the Germans are for the most part negative and still dominated by images of the Third Reich and the Second World War. It has even been suggested that ,kraut-bashing' is the only form of racism in Britain which is still considered socially acceptable (Wittlinger 2004:453).

Neben dem zweiten Weltkrieg wurden Einstellungen zu Deutschland und stereotypische Darstellungen der Deutschen in Großbritannien (und wahrscheinlich nicht nur dort) auch von anderen Faktoren geprägt: Deutschlands Wirtschaftswunder nach der Niederlage bzw. die neue politische und wirtschaftliche Macht Westdeutschlands in der Zeit von Margaret Thatcher; deutsche sportliche Leistungen (insbesondere was Fußball betrifft); die gegenwärtige Rolle Deutschlands in der Europäischen Union (deutschenfeindliche Argumente, welche die Gefahr einer deutschen Dominanz in Europa hervorheben, zählen zweifellos zu den wichtigsten innerhalb der Kampagne gegen die Europäische Union in Großbritannien, die 
2016 auf den Sieg des „leave“ beim britischen Referendum für den Austritt aus der EU hinausgelaufen ist). ${ }^{27}$

Bei einer Analyse der britischen Presse sticht die Anwendung stereotyper, nationalsozialismusbezogener Bilder von Deutschland deutlich heraus. Grix und Lacroix (2006), die eine derartige Analyse geliefert haben, weisen darauf hin, dass in der britischen Gesellschaft kontinuierlich Stereotypen über die Deutschen existieren, weil ,[t]he maintenance of stereotypes in the national psyche is kept up daily by the print and television media, with the latter devoting many hours to war-related documentaries and films“" (2006:374). Im Rahmen dieser Analyse wurde festgestellt, dass ,[a]lmost half (48\%) of the total pool of stereotypical portrayals of Germany were negative in nature ${ }^{28}$, compared with $32 \%$ neutral and $20 \%$ of a more positive nature" (Grix/Lacroix 2006:381). Dabei wurden acht besonders präsente Stereotypen über Deutschland und die Deutschen identifiziert: 1) „War prone“; 2) ,engine or leader of the $\mathrm{EU} “ ; 3)$ „German-British football rivalry“; 4) „highly intellectual, educated or creative“ ${ }^{\prime 29}$; 5) „German cars of engineering"; 6) „German companies hindering or taking over British ones“; 7) „controlled, restricted or highly legislated economy or society“; 8) „organised, punctual or boring" (Grix/Lacroix 2006:379). Die Parallelen sowohl mit den Stereotypen aus Abschnitt 2 als auch mit den Kulturstandards aus Abschnitt 3 dieses Beitrags sind unbestreitbar. Das Stereotyp der Deutschen als kriegsgeneigt wird im Kontext der Recherche von Grix \& Lacroix als ,a tendency for armed conflict, the use of violence or hostility (this also includes stereotypical references to Nazis or Nazism)" definiert (Grix/Lacroix 2006:378). Es ist leicht, zu sehen, dass sich diese mit Deutschland und mit den Deutschen verbundenen Konnotationen auf die deutsche Sprache übertragen. Relevant ist in diesem Sinne das folgende Beispiel eines Ausschnitts aus dem „Guardian“:

Why would you use a dash of German? Naturally, to fix on something dominant, domineering, muscle-flexing. German is used in submission to that which cannot be resisted, as in ,Vorsprung durch Technik' (no getting away from it), the longest phrase of German that most Britons will ever know. Now English has turned to this language to replace ,super' with a more imperious modern version. ,Uber' [sic!] is the uber-suffix [sic!] for anyone pretending

\footnotetext{
27 Detaillierter dazu Wittlinger (2004); Paterson (2001); Vasagar (2003); Beevor/Brockes (1999); Kershaw (2003).

28 Unter den vorkommenden Adjektiven zu den Deutschen: ,,arid“, „tidy-minded“, ,arrogant“, ,,suppressive“, „legalistic“, „ruthless“, „,dull“, ,,hierarchical“" and „,inflexible“ (Grix/Lacroix 2006:383).

29 Dieses vierte Stereotyp könnte einen Hinweis darstellen, dass die positiven Konnotationen der Zeit der „Dichter und Denker“ nach den Weltkriegen nicht komplett verschwunden sind. Dabei zeigt die Studie von Grix und Lacroix aber, dass derartige positive Stereotypisierungen in der in Betracht bezogenen Presse nur auf 20\% kommen.
} 
to sharp-eyed cultural comment (zit. n. Grix/Lacroix 2006:383; Formatierung von d. Verf. adaptiert).

Bemerkenswert ist die linguistische Wendung am Ende dieses Zitats, wo auf die Verbindung zwischen Imperialismus und deutscher Sprache hingewiesen wird. In der Studie von Grix und Lacroix wird dann noch eine „case study“ über das Stereotyp von Deutschland als ,engine or leader of the EU“ vorgestellt (vgl. Grix/ Lacroix 2006:385ff.). Diese spezifischere Analyse bringt zur Geltung, wie tief auch dieses Stereotyp mit den Ereignissen des Zweiten Weltkriegs verbunden ist. Die auf den Nationalsozialismus bezogenen Vorstellungen über die Deutschen scheinen einen starken Einfluss auf die britische Wahrnehmung der deutschen Rolle in der EU zu haben: „British print press coverage of Germany in the context of the EU can be seen as an extension of the warrelated stereotype. The sense conveyed is that Germany is attempting to achieve by economic means what it failed to achieve by military means" (Grix/Lacroix 2006:387). Eine Widerspiegelung dieser nicht nur in Großbritannien verbreiteten Idee weisen, einmal mehr, die vielen Memes über das Thema, wie dies beispielsweise in Abbildung $6^{30} \mathrm{zu}$ sehen ist.

\subsection{Modernes aus Print und virtuellen Räumen}

Wie sehen typische Ausdrücke bzw. Realisierungen der beliebtesten Stereotype über das Deutsche heutzutage aus? Ein humorvolles Beispiel ${ }^{31}$ kommt vom irischen Stand-up-Komiker Dylan Moran in der Sendung „Like, Totally“ (2006): „It's a disgusting language, nobody should ever speak it [...] you couldn't speak German 'cause it's a horrible sound, it sounds like a typewriter eating tinfoil being kicked down the stairs. Somebody is talking to you in German [...], you think, what is happening to you from behind, how can we make it stop?".32

Ein weiterer moderner, repräsentativer Fall sind natürlich die bereits besprochenen Memes, von denen ich im Laufe dieses Beitrags bereits mehrere Versionen aufgeführt habe. Bei diesen ging es um den Klang der Sprache, aber in dem Meme in der Abbildung 7 sieht das etwas anders aus. Hier können wir ahnen, dass nicht nur der Klang der Sprache eine Rolle in der deutlich negativen Darstellung des Deutschen spielt. So scheint z. B. die Gegenüberstellung des „langen“ deutschen Wortes Nasennebenhöhlenentzündung (26 Buchstaben) und der deutlich kürzeren Übersetzungen in anderen Sprachen ( 8 bis 9 Buchstaben) auf den angeblich höheren Schwierigkeitsgrad ${ }^{33}$ der deutschen Sprache hinzuweisen. Weiterhin wird hier

\footnotetext{
${ }^{30}$ Hier wird Deutschland von dem im Netz sogenannten „Germany ball“ verkörpert.

31 Für weitere Beispiele vgl. Brunner (2014).

$32 \mathrm{https} / /$ www.youtube.com/watch?v=IoLIU2NI66w. Zugriff am 10.6.2020.

33 Mit Schwierigkeitsgrad ist hier die empfundene Schwierigkeit gemeint, eine bestimmte Sprache zu lernen.
} 


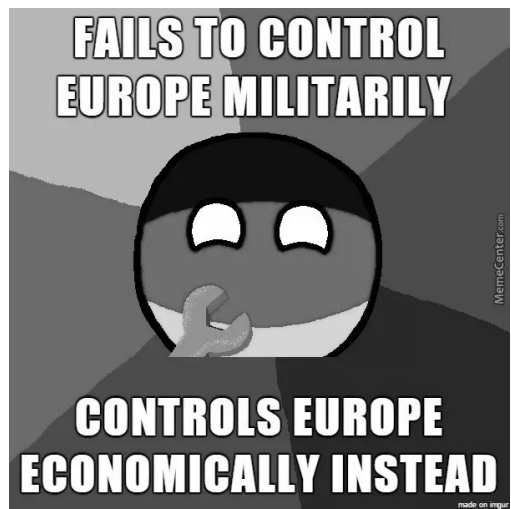

Abbildung 6: Germanyball-Meme, https://www.memecenter.com/fun/4945307/german-efficiency. Zugriff am 17.9.2020.

das Deutsche durch die Auswahl der anderen Sprachen von diesen isoliert: Die Sprachen, die zum Vergleich präsentiert werden, verwenden für diese Entzündung eine Bezeichnung lateinischen Ursprungs ${ }^{34}$ (übrigens auch das Englische mit seinen germanischen und romanischen Wurzeln), die sich daher ähneln oder sogar gleichen, während die deutsche Bezeichnung durch den völlig unterschiedlichen Aufbau heraussticht, was anders wäre, wenn man z. B. weitere germanische Sprachen heranzieht. ${ }^{35}$ Aussagekräftig für die Basis der Bewertung ist außerdem die Beschreibung, welche die Autor*innen des Beitrags als Begleitung für das Bild wählen: „Why I decided not to learn German!“. Das Meme ist also ein sehr gutes Beispiel einer Realisierung des im Abschnitt 2 identifizierten Stereotyps, dass Deutsch eine schwere Sprache sei. Derartige Memes entsprechen zudem manchen Videos auf YouTube, wie das beliebte „How German Sounds Compared To Other Languages“ ${ }^{\star 36}$, wo einige Begriffe jeweils auf Französisch, Englisch, Italienisch, Spanisch und Deutsch ausgesprochen werden. Alle Beteiligten werden sehr stereotyp dargestellt, dabei ist aber der deutsche Sprecher hier offensichtlich die Hauptfigur und trägt das deutsche Wort jedes Mal übertrieben aggressiv und energisch vor.

Die Firma „Big Seven Media“ ist dieses Thema etwas systematischer angegangen, indem sie eine Rangliste der „Top 50 Sexiest Accents In The World“37 veröffentlicht hat. Auf der ersten Stelle finden wir das in Neuseeland gesprochene Englisch (Kiwi), während sich das Deutsche auf Platz 46 befindet. Auf

\footnotetext{
34 Dabei existiert das Wort Sinusitis auch im Deutschen und gehört laut Duden sogar zu einer etwas höheren Häufigkeitsklasse als das Synonym Nasennebenhöhlenentzündung (Bibliographisches Institut GmbH 2020).

35 Vgl. z. B. das dänische Bihulebetcendelse oder das niederländische Bijholteontsteking.

${ }^{36}$ https://www.youtube.com/watch?v=jo0Hsx-yHiI. Zugriff am 10.6.2020.

37 https://bigseventravel.com/2019/04/worlds-sexiest-accent/. Zugriff am 10.6.2020.
} 


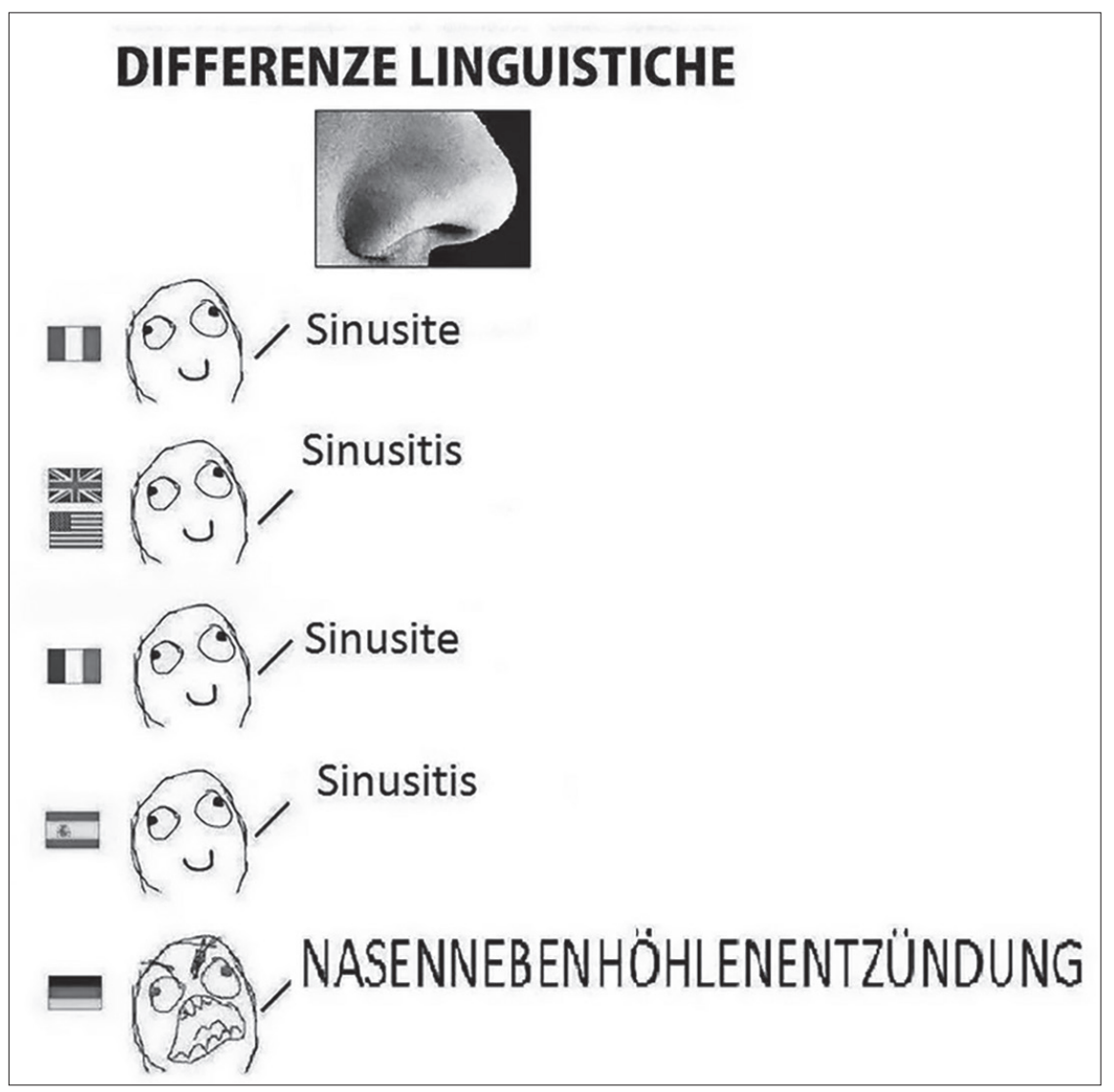

Abbildung 7: Nasennebenhöhlenentzündung-Meme, https://www.pinterest.com/pin/44149356969 1288055/. Zugriff am 17.9.2020.

Platz 4 ist das Italienische, auf Platz 7 das Französische und auf Platz 12 das "Queen Englisch“ (alle Sprachvarietäten, die üblicherweise als angenehm bzw. schön gelten). ${ }^{38}$ Vielsagend sind vor allem die kurzen Begründungen, die für die Position der verschiedenen Sprachen in der Liste angegeben werden. Über das Deutsche heißt es: „Harsh at times, yet super clear“. Wenn wir an das Modell aus Abschnitt 2 in diesem Beitrag zurückdenken, sehen wir hier wieder zwei der dort identifizierten Stereotype: das von der Härte und das von der Genauigkeit bzw. Gründlichkeit.

38 Die Rangliste hat sich aus einer Umfrage ergeben, an der 8500 Teilnehmer*innen aus 60 verschiedenen Ländern teilgenommen haben sollen (https://www.independent.co.uk/life-style/sexiestaccent-attractive-new-zealand-voice-ranking-a8891441.html - Zugriff am 10.6.2020). Mehr Informationen über die Entwicklung und Durchführung der Umfrage stellt die Internetseite nicht zur Verfügung (auf eine direkte Nachfrage gab es ebenfalls keine Antwort), weshalb diese Umfrage in unserem Rahmen keinen statistischen Wert aufweisen kann. 


\section{Schluss und Ausblick}

Die Fremdstereotype über die deutsche Sprache, die im Abschnitt 2 identifiziert wurden, haben sich in der darauffolgenden Analyse weitestgehend bestätigt: Für bildliche und sprachliche Realisierungen dieser Stereotype konnten sowohl im Netz als auch in der Literatur zahlreiche Beispiele aufgefunden werden (wobei hier nur eine Auswahl davon präsentiert wurde). Diese stereotypischen Meinungen waren auf der einen Seite auf die Sprache und auf der anderen Seite auf die Sprecher*innen gerichtet und haben sich auf die Aspekte der Didaktik, der Ästhetik und des Sprachverhaltens bezogen. Nach diesen (überwiegend negativen) Stereotypen sei die deutsche Sprache grundsätzlich schwierig, nützlich, unschön, hart und mit einem dominanten und präzisen (bis zu pedanten) Sprachverhalten verbunden. Von der Zeit, wo die Deutschen als ein Volk der Dichter und Denker hochgeschätzt wurden, bis zur dunklen Zeit der Weltkriege und zur modernen Europäischen Union, wurde die Außensicht über Deutschland und die Deutschen durch einzelne historische Ereignisse stark geprägt. Obwohl die genauen Gründe, die hinter der Wahrnehmung von Sprachen stecken, noch unzureichend erforscht sind und eine tiefere sprachwissenschaftliche Auseinandersetzung mit dem Thema verdienen würden, stellen gesellschaftliche (und darunter historische) Konnotationen ohne Zweifel einen schwerwiegenden Einflussfaktor dar. Dementsprechend konnte durch die vorliegende Analyse eine deutliche Verbindung zwischen land- bzw. volksbezogenen Stereotypen und sprachbezogenen Stereotypen hervorgehoben werden.

Ja, die negativen Stereotype scheinen zu überwiegen, das ist bei Stereotypen aber meistens so (ist es denn nicht so, dass das Negative immer eher als das Positive auffällt?). Sollen deswegen die Liebhaber*innen der deutschen Sprache verzweifeln? Wahrscheinlich nicht. Wenn es auch am Ende tatsächlich die Mehrheit der Nichtdeutschen ist, die einen negativen Eindruck von der deutschen Sprache hat (was noch nicht ausschlaggebend bewiesen ist), ist die positive Meinung der Minderheit deswegen nicht wertlos. Man könnte auch die Sicht des Komponisten Arnold Schönbergvertreten, „[d]enn wenn es Kunst ist, ist sie nicht für alle, und wenn sie für alle ist, ist sie keine Kunst“" (1995:53).

\section{Literatur}

BARnes Frank C., 1918, Shall German Be Dropped from Our Schools?, in: The Modern Language Journal 2, S. 187-202.

BeEvor Antony / Brockers Emma, 1999, Tommy and Jerry, in: The Guardian v. 16.2.

BERTHELE Raphael, 2010, Investigations into the folk's mental models of linguistic varieties, in: Geeraerts D./Kristiansen G./Peirsman Y. (Hrsg.), Advances in Cognitive Sociolinguistics, Berlin/ New York, S. 265-290. 
Bibliographisches Institut GmbH, 2020, Häufigkeit, https://www.duden.de/hilfe/haeufigkeit. Zugriff am 19.6.2020.

BoEHLICH Walter, 1964, Sind wir noch ein Volk der Dichter und Denker?, https://www.zeit.de/1964/07/ sind-wir-noch-ein-volk-der-dichter-und-denker. Zugriff am 19.6.2020.

Borst Arno, 1966, Wie sprach Kaiser Karl mit seinem Pferd?: Fremde Leute, fremde Zungen - Am Beispiel einer berühmten Anekdote, http://www.zeit.de/1966/48/wie-sprach-kaiser-karl-mitseinem-pferd. Zugriff am 19.6.2020.

Bounours Dominique, 1708, Les entretiens d'Ariste et d'Eugène, Amsterdam.

BRUNNER Bernd, 2014, „Wie eine Schreibmaschine, die Alufolie frisst“: Der Klang der deutschen Sprache, https://www.deutschlandfunk.de/der-klang-der-deutschen-sprache-wie-eine-schreibmaschine.1184.de.html?dram:article_id=307163. Zugriff am 19.6.2020.

Bulwer Lytton Edward, 1866, Ernest Maltravers; or, the Eleusinia, Philadelphia.

De Stä̈L-Holstein Anne Louise Germaine, 1815, Deutschland: aus dem Französischen übersetzt, Mäcken.

Delumeau Jean, ${ }^{1} 2000$, History of paradise: The Garden of Eden in myth and tradition, Urbana.

DePenheuer Otto / PeIFER Klaus-Nikolaus, 2008, Geistiges Eigentum: Schutzrecht oder Ausbeutungstitel?, Berlin/Heidelberg.

EICHINGER Ludwig M., 2017, Was macht Stickel?, in: Itakura U./Dąbrowska-Burkhardt J./Eichinger L.M. (Hrsg.), Deutsch: lokal - regional - global, Tübingen.

FABRICI Girolamo d'Acquapendente, 1601, De locutione et eius instrumentis: liber a Ioanne Vrsino, Venetiis.

Fitz-Gerald John D., 1918, National Aspects of Modern Language Teaching in the Present Emergency, in: The Modern Language Journal 3, S. 49-62.

Froude James Anthony / Tulloch John / Carlyle Thomas, 1860, Fraser's Magazine.

Gärtig Anne-Kathrin / Plewnia Albrecht / Rothe Astrid, 2010, Wie Menschen in Deutschland über Sprache denken: Ergebnisse einer bundesweiten Repräsentativerhebung zu aktuellen Spracheinstellungen, Mannheim.

Giles Howard / Bourhis Richard / Lewis Alan / Trudgill Peter, 1974, The imposed norm hypothesis: A validation, in: Quarterly Journal of Speech 60, S. 405-410.

Grody Miles H., 1918, The German language in our schools, in: Educational Review 56, S. 257-263.

Grix Jonathan / LaCroIX Chantal, 2006, Constructing Germany's Image in the British Press: An Empirical Analysis of Stereotypical Reporting on Germany, in: Journal of Contemporary European Studies 14, S. 373-392.

HANDSCHIN Charles Hart, 1913, The teaching of modern languages in the United States, Washington, DC.

HeINE Heinrich, 1876, Über Deutschland, Hamburg.

Herder Johann Gottfried, 1803, Adrastea, Leipzig.

Hofstadter Douglas R., ${ }^{20} 1999$, Gödel, Escher, Bach: An eternal golden braid, New York.

JONES William Jervis, 1999, Images of Language, Amsterdam.

KaINZ Friedrich, 1974, Klassik und Romantik, in: Maurer F./Rupp H. (Hrsg.), Deutsche Wortgeschichte, Berlin/New York, S. 245-491.

Kershaw Ian, 2003, The thing about Hitler, in: The Guardian v. 29.1.

Kraus Karl, 1967, Die dritte Walpurgisnacht, München.

Levi Primo, 2010, Ist das ein Mensch?: Ein autobiographischer Bericht, München.

LuebKe Frederick C., 1980, Legal Restrictions on Foreign Languages in the Great Plains States, 1917-1923, in: Schach P. (Hrsg.), Languages in conflict: Linguistic acculturation on the Great Plains, Lincoln, S. 1-19.

MCGARTY Craig / Yzerbyt Vincent / Spears Russell, 2002, Stereotypes as explanations: The formation of meaningful beliefs about social groups, London/New York. 
Menasse Robert, 2004, Lob der deutschen Sprache, Rede zur Eröffnung des Internationalen Germanistenkongresses am 12.09.2004 in München, http://www.detlev-mahnert.de/Lob_der_deutschen_Sprache.html. Zugriff am 15.10.2020.

MenZel Wolfgang, 21836, Die deutsche Literatur, Stuttgart.

Milling Hanna, 2010, Das Fremde im Spiegel des Selbst: Deutschland seit dem Mauerfall aus Sicht französischer, italienischer und spanischer Deutschlandexperten, Berlin.

Moran Dylan, 2006, Dylan Moran on Germany.

Müller-Marein Josef, 1963, Das Pferde-Plagiat, https://www.zeit.de/1963/09/das-pferde-plagiat. Zugriff am 19.6.2020.

PATERSON William, 2001, Britain and the Berlin Republic: Between Ambivalence and Emulation, in: German Politics 10, S. 201-223.

Pavlenko Aneta, 2003, 'Language of the Enemy': Foreign Language Education and National Identity, in: International Journal of Bilingual Education and Bilingualism 6, S. 313-331.

PeAcock Thomas Love, 1861, Gryll grange, London.

Primo Levi, 1979, Se questo è un uomo, Milano.

PURSCHKE Christoph, 2011, Regionalsprache und Hörerurteil: Grundzüge einer perzeptiven Variationslinguistik, Stuttgart.

RusCONI Gian Enrico, 2001a, Etappen einer Erfolgsgeschichte. Ein halbes Jahrhundert GoetheInstitute in Italien, in: Kaußen H. (Hrsg.), Murnau, Manila, Minsk-50 Jahre Goethe-Institut, München.

RUSCONI Gian Enrico, 2001b, http://www.goethe.de/ins/it/de/lp/uun/102523.html. Zugriff am 1.7.2016. SCHILLER Friedrich, 1902, Deutsche Größe, Weimar.

ScHönberg Arnold, 1995, Neue Musik, veraltete Musik, Stil und Gedanke, in: Schönberg A./Vojtech I. (Hrsg.), Stil und Gedanke, Frankfurt am Main, S. 40-53.

Scholl-Machl Sylvia, 2002, Die Deutschen - Wir Deutsche: Mit 2 Abbildungen und einer Tabelle / Fremdwahrnehmung und Selbstsicht im Berufsleben, Göttingen.

Stickel Gerhard, 2003, Zur deutschen Sprache: die Innensicht der Außensicht, in: Stickel G. (Hrsg.), Deutsch von außen, Berlin, S. 1-14.

SwIFT Jonathan, 1999, Gulliver's Travels: into Several Remote Nations of the World, London.

Thomas Alexander, 1999, Kultur als Orientierungssystem und Kulturstandards als Bauteile, in: Board of the Institute for Migration Research and Intercultural Studies (Hrsg.), IMIS-Beiträge, Bramsche.

Traub Rainer, 2007, Unter der Fahne der Wahrheit und Schönheit, http://www.spiegel.de/spiegel/sp iegelspecialgeschichte/d-50620300.html. Zugriff am 19.6.2020.

Trudgill Peter / Giles Howard, 1976, Sociolinguistics and Linguistic Value Judgements: Correctness, Adequacy and Aesthetics, Trier.

TwaIn Mark, 2016, The Awful German Language, Berlin.

VASAGar Jeevan, 2003, Don't mention the war to the Germans . . . the Gulf war, in: The Guardian v. 4.7.

Von GoETHE Johann Wolfgang, 2005, Faust, Milano.

WiLEy Terrence G., 1998, The imposition of World War I era: English-Only policies and the fate of German in North America, in: Ricento T./Burnaby B. (Hrsg.), Language and politics in the United States and Canada: Myths and realities, Mahwah N.J., S. 211-241.

WitTlinger Ruth, 2004, Perceptions of Germany and the Germans in Post-war Britain, in: Journal of Multilingual and Multicultural Development 25, S. 453-465.

Zeydel Edwin H., 1964, The Teaching of German in the United States from Colonial Times to the Present, in: The German Quarterly 37, S. 315-392. 


\section{Abbildungsverzeichnis}

Abb. 1 (adaptiert): How to say butterfly-Meme, https://www.memedroid.com/memes/detail/640043. Zugriff am 17.9.2020.

Abb. 2 (adaptiert):Unterscheidungsvermögen-Meme,https://hugelol.com/lol/120122.Zugriffam17.9. 2020.

Abb. 3 (adaptiert): How Germans see Germans taking to each other-Meme, https://www.memecenter. $\mathrm{com} /$ fun/1791117/how-we-see-german-amp-039-s-talking. Zugriff am 17.9.2020.

Abb. 4: Darstellung der Meinungen aus der Stickel-Umfrage.

Abb. 5: Darstellung der Korrespondenz zwischen Meinungen aus der Stickel-Umfrage und deutschen Kulturstandards.

Abb. 6 (adaptiert): Germanyball-Meme, https://www.memecenter.com/fun/4945307/german-efficiency. Zugriff am 17.9.2020.

Abb. 7 (adaptiert): Nasennebenhöhlenentzündung-Meme, https://www.pinterest.com/pin/44149356 9691288055/. Zugriff am 17.9.2020.

\section{Outside-stereotypes about the German language and what hides behind them}

The German language is (stereo)typically viewed as a language that is both hard to learn and harsh to the ear, as well as useful and (sometimes overly) precise. Considering a few linguistic studies but focusing on layman manifestations from different countries found in literature, press, television and social networks, the author analyses these stereotypes as to their spread, connection to German culture, possible origins and social connotations. Following a qualitative approach, the analysis highlights how the outside view of Germany and the Germans has been shaped by specific historical events, from the era of the Germans as a people of poets and thinkers to the two World Wars and the modern EU. Finally, the analysis allows the identification of a clear connection between Germany/ Germans-related and German-related stereotypes.

Keywords: German language, linguistic stereotypes, social connotations, language attitudes, folk linguistics. 